\title{
Non-Viral Bone Morphogenetic Protein 2 Transfection of Rat Dental Pulp Stem Cells Using Calcium Phosphate Nanoparticles as Carriers
}

\author{
XUECHAO YANG, D.D.S., Ph.D., ${ }^{1,2}$ X. FRANK WALBOOMERS, Ph.D., ${ }^{1}$ \\ JULIETTE VAN DEN DOLDER, Ph.D., ${ }^{1}$ FANG YANG, Ph.D., ${ }^{1}$ ZHUAN BIAN, D.D.S., Ph.D., \\ MINGWEN FAN, D.D.S., Ph.D., ${ }^{2}$ and JOHN A. JANSEN, D.D.S., Ph.D. ${ }^{1}$
}

\begin{abstract}
Calcium phosphate nanoparticles have shown potential as non-viral vectors for gene delivery. The aim of this study was to induce bone morphogenetic protein (Bmp) 2 transfection in rat dental pulp stem cells using calcium phosphate nanoparticles as a gene vector and then to evaluate the efficiency and bioactivity of the transfection. We also intended to investigate the behavior of transfected cells when seeded on 3dimensional titanium fiber mesh scaffolds. Nanoparticles of calcium phosphate encapsulating plasmid deoxyribonucleic acid (DNA) (plasmid enhanced green fluorescent protein-BMP2) were prepared. Then, STRO-1-selected rat dental pulp stem cells were transfected using these nanoparticles. Transfection and bioactivity of the secreted BMP2 were examined. Thereafter, the transfected cells were cultured on a fibrous titanium mesh. The cultures were investigated using scanning electron microscipy and evaluated for cell proliferation, alkaline phosphatase activity and calcium content. Finally, real-time polymerase chain reaction was performed for odontogenesis-related gene expression. The results showed that the size of the DNA-loaded particles was approximately $100 \mathrm{~nm}$ in diameter. Nanoparticles could protect the DNA encapsulated inside from external DNase and release the loaded DNA in a low-acid environment (pH 3.0). In vitro, nanoparticle transfection was shown to be effective and to accelerate or promote the odontogenic differentiation of rat dental pulp stem cells when cultured in the 3-dimensional scaffolds. Based on our results, plasmid DNA-loaded calcium phosphate nanoparticles appear to be an effective non-viral vector for gene delivery and functioned well for odontogenic differentiation through Bmp2 transfection.
\end{abstract}

\section{INTRODUCTION}

$\mathbf{I}^{2}$ NISSUE ENGINEERING, GENE THERAPY is used as a means of delivering genes locally to somatic cells, encoding for proteins like growth factors, transcription factors, or extracellular matrix molecules with a resulting therapeutic effect. The applied genes can stimulate or induce a natural biological process by expressing bioactive molecules that are involved in the regenerative response of the tissue. ${ }^{1}$ Gene therapy can be a valuable tool to avoid the limitations of local delivery of growth factors, including short half-life, large dose requirement, high cost, need for repeated applications, and poor distribution. ${ }^{2}$ In our previous study, an adenoviral-mediated human bone morphogenetic protein (Bmp) 2 gene was transfected into STRO-1-selected rat dental pulp stem cells (DPSCs) and showed its ability to promote odontogenic action effectively. ${ }^{3}$ However, the clinical application of an adenovirus is not advisable, because it

\footnotetext{
${ }^{1}$ Radboud University Nijmegen Medical Centre, Department of Periodontology and Biomaterials, Nijmegen, The Netherlands.

${ }^{2}$ Key Lab for Oral Biomedical Engineering of Ministry of Education, School and Hospital of Stomatology, Wuhan University, Hubei Province, People's Republic of China.
} 
results in endogenous recombination, oncogenic effects, and toxic immunological reactions, leading to potential serious complications. ${ }^{4,5}$ For this reason, use of a number of nonviral vectors have been explored. The application of inorganic nanoparticles with entrapped deoxyribonucleic acid (DNA) is a new field for non-viral transfection and is expected to overcome the limitations mentioned above. The advantages of these vectors are the ease of manufacture, low cost, stability, low immunogenicity, and low risk for the plasmid to be inserted into the host cell genome. In the literature, plasmid DNA (pDNA)-loaded calcium phosphate nanoparticles have already been demonstrated to be highly efficient for transfection in the Hela cell line. 6,7 $^{\text {. }}$

The osteoinductive potential of recombinant human BMP2 (hBMP2) has already been confirmed in a clinical study. ${ }^{8}$ In our study, the recombinant human BMP2 gene was used for transfection because it can stimulate the differentiation of adult dental pulp cells into odontoblasts, and it also plays a role during the various stages of the formation of hard skeletal and dental tissue in bone and teeth. ${ }^{9-11}$ Furthermore, for our dental tissue-engineering purposes, DPSCs were used as these responding stem cells in nature. A hierarchy of DPSCs causes the variable behavior of these cells in vitro and results in a low therapeutic potential in vivo. ${ }^{12,13}$ STRO- ${ }^{+}$DPSCs were chosen because selection with this marker increased the ability of osteo- and odontogenic differentiation, as well as development toward other differentiation pathways. ${ }^{3,14,15}$

In this study, we intended to transfect STRO-1-selected rat DPSCs with pDNA entrapped in calcium phosphate nanoparticles. We hypothesized that calcium phosphate nanoparticles would result in a relatively high transfection efficacy and that the secreted BMP2 would be effective in odontoinductive action.

\section{MATERIAL AND METHODS}

\section{Preparation of pDNA-loaded calcium phosphate nanoparticles}

A recombinant green fluorescent eukaryotic cell expressing the plasmid-carrying $h B m p 2$ gene (plasmid enhanced green fluorescent protein-BMP2 (pEGFP-BMP2), was used in the current study. The preparation of pDNA-loaded calcium phosphate nanoparticles followed the protocol of Bisht et $a$. $^{7}$ The basic method was as follows; 0.1 M bis (2ethylhexyl) sulphosuccinate (Aerosol ${ }^{\circledR}$-OT, AOT), solution in hexane (Sigma, St. Louis, MO) was prepared. In $25 \mathrm{~mL}$ of AOT in hexane, $70-\mu \mathrm{L}$ of aqueous solution of $1.36 \mathrm{M}$ calcium chloride and aqueous solution of $2.94 \mu \mathrm{g}$ of pDNA were added by continuous stirring overnight to form a microemulsion "A." In another $25 \mathrm{~mL}$ of AOT solution, $50 \mu \mathrm{L}$ of $0.2 \mathrm{M}$ Tris-hydrochloric acid buffer ( $\mathrm{pH} 7.4$ ), $70 \mu \mathrm{L}$ of aqueous solution of $0.35 \mathrm{M}$ dibasic sodium phosphate, and an aqueous solution of $2.94 \mu \mathrm{g}$ of pDNA were dissolved by continuous stirring overnight to form micro-emulsion "B."
Before stirring both micro-emulsions, excess water was added to make the total volume of $450 \mu \mathrm{L}$ to adjust the molar ratio of water to AOT at 10 to 1 . Then, micro-emulsion "B" was added to "A" at an extremely slow rate $(5 \mathrm{~mL}$ per min) with continuous stirring at $4{ }^{\circ} \mathrm{C}$ and was kept in the same condition overnight. Subsequently, hexane was carefully removed from the resulting solution after centrifuging $30 \mathrm{~min}$ at 5000 revolutions per min at $4{ }^{\circ} \mathrm{C}$. The sediment was dissolved in $10 \mathrm{~mL}$ of absolute ethanol by vortexing, followed again by centrifugation. After 3 such cycles, the pelleted nanoparticles were dispersed in Milli-Q water at $4^{\circ} \mathrm{C}$ by vortexing. Finally, the dispersed nanoparticles were lyophilized to dry powder (yield $\sim 1 \mathrm{mg}$ ).

\section{Morphology and characteristics of the nanoparticles}

Transmission electron microscopy. To evaluate the size of the nanoparticles, 1 drop of aqueous dispersion of the particles was put on a Forvmar-coated copper grid and air dried at room temperature. The dried grid was examined under a JEOL (Tokyo, Japan) 1101 transmission electron microscope (TEM) coupled to digital image analysis software. Approximately 25 particles were measured.

Gel electrophoresis. To check the protection of DNA in the nanoparticles from external DNase degradation, $20 \mu \mathrm{L}$ of aqueous dispersion of calcium phosphate/DNA nanoparticles (containing $1 \mu \mathrm{g}$ of $\mathrm{pDNA}$ ) prepared by the above method was incubated with $5 \mu \mathrm{L}$ of DNaseI $(5 \mathrm{mg} / \mathrm{mL})$ at $37^{\circ} \mathrm{C}$ for different time intervals $(0,0.5$, and $1 \mathrm{~h})$ and then loaded in $1 \%$ agarose gel. As positive and negative controls, $10 \mu \mathrm{L}$ of free pDNA was loaded alone or after incubation with DNaseI.

Entrapment efficiency. The entrapment efficiency of DNA attached to the nanoparticles was evaluated by dissolving the particles in acidic buffer ( $\mathrm{pH}$ 3.0) and incubating overnight. The amount of pDNA released from the nanoparticles ([pDNA]r) was estimated spectrophotometrically by measuring the optical density (OD) at $\lambda=260 \mathrm{~nm}$. The entrapment efficiency was then calculated from the amount of DNA originally added in the solution ([pDNA $\left.]_{0}\right)$, using the equation $\mathrm{E} \%=[\mathrm{pDNA}] \mathrm{r} /[\mathrm{pDNA}]_{0} \times 100$.

\section{In vitro transfection studies}

Cell culture. Rat DPSCs (10th passage) were selected on the stem cell marker STRO-1 using fluorescence-activated cell sorting, as described previously, ${ }^{17}$ and expanded in minimal essential medium-alpha ( $\alpha$-MEM; GIBCO-BRL, Breda, The Netherlands) supplemented with $10 \%$ fetal calf serum (FCS; GIBCO-BRL) and $50 \mu \mathrm{g} / \mathrm{mL}$ of gentamycin (Sigma).

Determination of transfection efficiency. The cells were seeded in 24-well plates at a density of $2 \times 10^{4}$ cells in $1 \mathrm{~mL}$ of culture medium per well. Upon reaching $80 \%$ confluency, 
cells were used for transfection. Then $0.3 \mathrm{~mL}$ of pEGFPBMP2-loaded calcium phosphate nanoparticles in $\alpha$-MEM without FCS and antibiotics (total amount of pDNA $\sim 0.5 \mu \mathrm{g}$ ) was added to each well. After incubation at $37^{\circ} \mathrm{C}$ for $4 \mathrm{~h}$, the transfection complex was replaced with fresh $\alpha$ MEM containing 10\% FCS and antibiotics. Exactly $48 \mathrm{~h}$ post-transfection, cells were detached to detect green fluorescence using fluorescence microscopy to confirm temporary transfection. Simultaneously, the supernatants were collected on days $2,4,6,8$, and 10 after transfection. Aliquots of $50 \mu \mathrm{L}$ of these samples were used to measure the concentration of recombinant hBMP2 by using a BMP2 enzyme-linked immunosorbent assay (ELISA) kit (Quantikine, R\&D Systems, Inc. Minneapolis, MN) $(n=6)$. Also, at days $1,4,8$, and 16 after transfection, the proliferation rates of the cells, as measured according to total DNA content $(n=6)$, were assessed using a Pico Green double-strand DNA quantification kit (Molecular Probes, Leiden, The Netherlands). ${ }^{16}$ The commercial transfection reagent Lipofectamine 2000 (Invitrogen, Gaithersburg, MD) was used as a control using the same cell density and incubation times.

The bioactivity of hBMP2 produced from transfected cells was assessed according to alkaline phosphatase (ALP) activity upregulation using the cell line W20-17, as reported previously. ${ }^{20}$ Briefly, $48 \mathrm{~h}$ post-transfection, the supernatants were collected and filtered. Then these conditioned media were transferred to $80 \%$ confluent W20-17 cells $(n=6)$. After an additional $48 \mathrm{~h}$ of incubation, a lysate was prepared to measure ALP activity of the W20-17 cells. ${ }^{17}$

\section{Three-dimensional scaffold culture}

Titanium fiber mesh (Bekenit, SaitamaKen, Japan) was used as a 3-dimensional (3-D) scaffold. The fiber diameter was $45 \mu \mathrm{m}$. The volumetric porosity of the total mesh was $86 \%$, and the weight was $400 \mathrm{~g} / \mathrm{m}^{2}$. A titanium fiber mesh sheet was cut into discs of $6 \mathrm{~mm}$ in diameter and $1 \mathrm{~mm}$ in thickness, and then scaffolds were sterilized using autoclaving.

Cell seeding. All scaffolds were pre-wetted in $\alpha$-MEM with $10 \%$ FCS and $50 \mu \mathrm{g} / \mathrm{mL}$ of gentamycin overnight. Forty-eight $\mathrm{h}$ post-transfection, STRO-1-selected DPSCs were seeded onto the scaffolds by incubating the scaffolds in a suspension of $1.0 \times 10^{6}$ cells $/ \mathrm{mL}$ while gently rotating at 6 revolutions per min for $3 \mathrm{~h}$. For every 6 scaffolds, $1 \mathrm{~mL}$ of cell suspension was used. Subsequently, the scaffolds were put in 24-well plates for culturing with $\alpha$-MEM supplemented with $10 \%$ FCS, $10 \mathrm{mM}$ sodium $\beta$-glycerophosphate, $10^{-8} \mathrm{M}$ dexamethasone, $50 \mu \mathrm{g} / \mathrm{mL}$ of L-ascorbic acid, and $50 \mu \mathrm{g} / \mathrm{mL}$ of gentamycin. The non-transfected cells were used as a control and cultured in the same osteogenic medium as above or in non-osteogenic medium ( $\alpha$-MEM supplemented with $10 \%$ FCS and $50 \mu \mathrm{g} / \mathrm{mL}$ of gentamycin.). Culture media were replaced at 3-day intervals.

Cell proliferation, ALP activity, and calcium content. On days $1,4,8,16$, and 24 post-seeding, the proliferation rates of cells, as measured according to total DNA content $(n=3)$, were assessed. The same samples as used above were also used to measure ALP activity. Twenty $\mu \mathrm{L}$ of 0.5 AMP buffer (Sigma) was added to $80 \mu \mathrm{L}$ of the samples or standards. Next, $100 \mu \mathrm{L}$ of substrate solution was added, and the mixtures were incubated at $37^{\circ} \mathrm{C}$ for $1 \mathrm{~h}$. ALP activity was measured at $405 \mathrm{~nm}$ using an ELISA microplate reader (Bio-Tek Instruments Inc., Winooski, VT). On days 4, 8, 16, and 24 after seeding, the calcium content of the samples $(n=3)$ was measured using the $o$-cresolphthalein complexone (Sigma) method. ${ }^{16}$

Scanning electron microscopy. On days 1, 8, and 24 after cell seeding, scaffolds were rinsed with filtered PBS, fixed in $2 \%$ glutaraldehyde for $5 \mathrm{~min}$, dehydrated in graded series of ethanol, and dried in tetramethylsilane (Merck, Darmstadt, Germany). Gold was sputtered on the samples just before SEM analysis. The specimens were examined using a JEOL $6310 \mathrm{SEM}$ at an accelerating voltage of $10 \mathrm{kV}$.

Real-time quantitative polymerase chain reaction. In a pilot study preceding the current experiment (data not shown), we found that the messenger ribonucleic acids (mRNAs) were active and detectable for 16 days and subsequently significantly descended to day 24 , so for the current study only, at days $1,4,8$, and 16 after cell seeding, total RNA of every sample was prepared with Qiagen RNEasy kit (Qiagen, Venlo, The Netherlands). Then, $1 \mu \mathrm{g}$ of total RNA was used to perform the reverse transcriptase (RT) reaction. The obtained complementary DNA was used as a template in PCR. The odontogenic differentiation of cells was monitored according to specific markers: ALP, collagen type I (Col I ), osteocalcin (OC), dentin sialophosphoprotein

Table 1. The Rat-Specific Primer Sequences Used for Real-Time Polymerase Chain Reaction

\begin{tabular}{|c|c|c|}
\hline & Sense primer & Antisense primer \\
\hline ALP & $5^{\prime}$-GCTTCACGGCATCCATGAG- $3^{\prime}$ & 5'-GAGGCATACGCCATGACGT-3 \\
\hline $\mathrm{OC}$ & 5'-GGCTTCCAGGACGCCTACA-3 & 5'-CATGCCCTAAACGGTGGTG-3' \\
\hline Col I & 5'-TGGAATCTTGGATGGTTTGGA-3' & $5^{\prime}$-GCTGTAAACGTGGAAGCAAGG-3' \\
\hline DSPP & $5^{\prime}$-CGGTCCCTCAGTTAGTC-3' & $5^{\prime}$-TACGTCCTCGCGTTCT-3' \\
\hline DMP-1 & 5'-CGTTCCTCTGGGGGCTGTCC-3' & 5'-CCGGGATCATCGCTCTGCATC-3' \\
\hline GAPDH & 5'-GAAGGGCTCATGACCACAGT- $3^{\prime}$ & 5'-GGATGCAGGGATGATGTTCT-3 \\
\hline
\end{tabular}


(DSPP), and dentin matrix protein 1 (DMP-1). Glyceraldehyde 3-phosphate dehydrogenase (GAPDH) was used as a housekeeping gene to normalize RNA expression (Table 1).

Real-time PCR was performed using SYBR Green PCR kit (Eurogentec, Liege, Belgium) and controlled in a spectrofluorometric thermal iCycler (Bio-Rad, Veenendaal, The Netherlands). After the real-time PCR run, the threshold cycle $(\mathrm{Ct})$ value showed how many PCR cycles were necessary to obtain a certain level of fluorescence. Amplification efficiency of different genes was determined relative to GAPDH as an internal control $(\Delta \mathrm{Ct}=\mathrm{Ct}$ gene $-\mathrm{Ct}$ GAPDH). The mRNA in each sample was calculated using the comparative $\Delta \Delta \mathrm{Ct}(\Delta \mathrm{Ct}$ gene $-\Delta \mathrm{Ct}$ control) value method. Each sample was assessed in triplicate.

\section{Statistical analysis}

Statistical significance was evaluated using one-way analysis of variance with post hoc Tukey testing. As indicated, $p<0.05$ was considered significant. Error bars represent means \pm standard deviations.

\section{RESULTS}

\section{pDNA-loaded calcium phosphate nanoparticles}

After continuous stirring overnight, the micro-emulsions "A" and "B" showed optically clear solutions. In the resulting solution (mixture of "A" and "B"), development of translucency implicated nanoparticle formation in the aqueous core of the micro-emulsion droplets. The TEM investigation revealed the formation of dense particles with a spherical morphology. When dispersed in water, the particle size was in the range of 75-100 nm in diameter (Fig. 1). The resistance of pDNA-loaded calcium phosphate complexes to

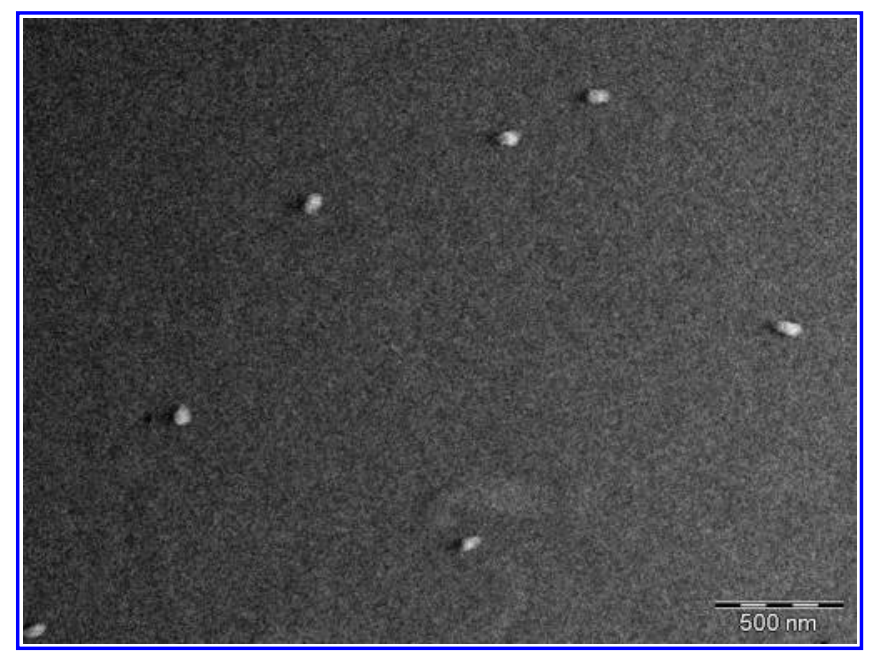

FIG. 1. Transmission electron micrograph of plasmid deoxyribonucleic acid-encapsulated calcium phosphate nanoparticles. external aggressive DNase attack was demonstrated using gel electrophoresis (Fig. 2). Although free pDNA could move to the usual position $(\sim 6 \mathrm{~kb})$ and could also be completely digested by external DNase, the plasmid-loaded complexes had hardly moved and stayed at the top of the lanes. One mg of pDNA-loaded nanoparticles, in which the original amount of applied DNA was $5.88 \mu \mathrm{g}$, was used to evaluate the entrapment efficiency of DNA inside the nanoparticles. After overnight incubation, the entrapment efficiency was calculated as $97.15 \% \pm 2.04 \%(n=4)$.

\section{In vitro transfection studies}

The successful transfection could be easily traced using green fluorescence protein (GFP). Forty-eighth posttreatment of nanoparticles, the DPSCs showed the expression of green fluorescence, visually demonstrating a similar positive rate to that of cells transfected using Lipofectamine 2000 (Fig. 3). The amount of hBMP2 secreted by transfected rat DPSCs was quantitated using ELISA (Fig. 4A). With Lipofectamine 2000 transfection, the cells produced significantly more hBMP2 on day 2 , although on days 8 and 10 , the expression of hBMP2 was significantly lower. The DNA results showed significantly higher cell numbers in the nanoparticle group for all time points except on day 16 , although the trend of proliferation was similar to the cells transfected with Lipofectamine 2000 (Fig. 4B). The bioactivity of hBMP2 was assessed according to the ability of conditioned media to upregulate the ALP activity of the BMP2-sensitive W20-17 cell line (Fig. 4C). The medium from rat DPSCs transfected with nanoparticles demonstrated significantly greater W20-17 ALP activity than in the control group of non-transfected cells $(p<0.01)$.

\section{3-D scaffold culture}

The cell proliferation rates are depicted in Figure 5A. Analysis of the data showed that the proliferation of nontransfected rat DPSCs in non-osteogenic medium was significantly faster than that of transfected cells and nontransfected rat DPSCs in osteogenic medium, although no significant differences were found between these latter 2 groups. The ALP activity of both cell groups cultured in osteogenic medium increased over 24 days, but transfected DPSCs showed significantly higher levels after day 4 than non-transfected cells (Fig. 5B). The non-transfected cells cultured in non-osteogenic medium always demonstrated significantly lower levels of ALP activity $(p<0.01)$ and only started to increase after day 8 . The calcium content increased significantly between days 8 and 24 for 2 cell groups cultured in osteogenic medium $(p<0.01)$, but the transfected cells showed significantly higher levels after day 16 than non-transfected cells $(p<0.01)$ (Fig. 5C).

SEM observation showed that cells already attached and spread on the surface of Ti-fibers after 1 day of seeding and proliferated along the culture. The rat DPSCs in non- 


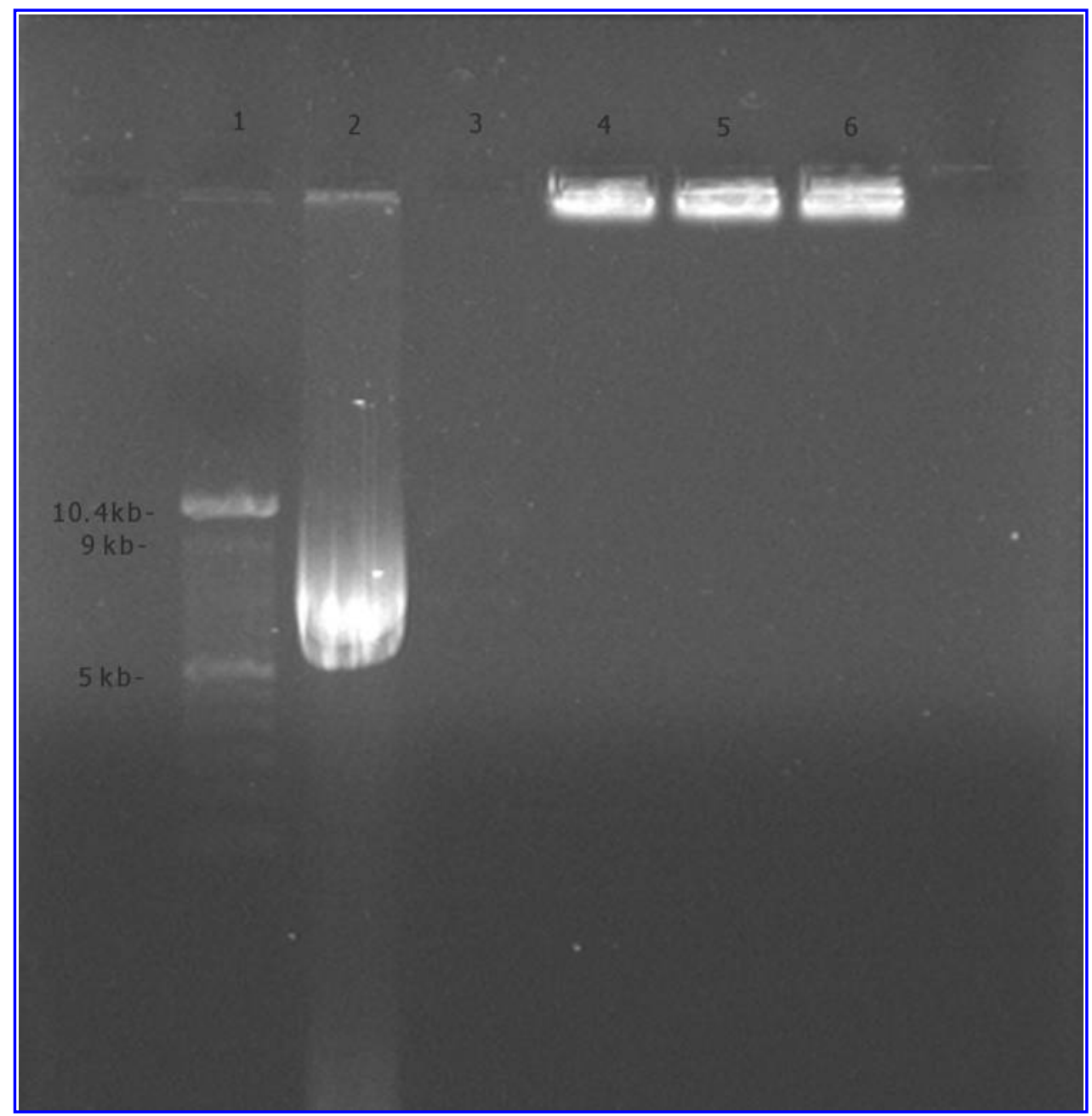

FIG. 2. Agarose gel (1\%) electrophoresis. Lane 1: Deoxyribonucleic acid (DNA) marker. Lane 2: free permuted enhanced green fluorescent protein-bone morphogenetic protein 2 (pEGFP-BMP2) DNA (6 kb). Lane 3: pEGFP-BMP2 DNA treated with DNase for 0.5 h. Lane 4: pEGFP-BMP2-loaded calcium phosphate nanoparticles. Lane 5: pEGFP-BMP2-loaded calcium phosphate nanoparticles treated with DNase for $0.5 \mathrm{~h}$. Lane 6: pEGFP-BMP2-loaded calcium phosphate nanoparticles treated with DNase for $1 \mathrm{~h}$.

osteogenic culture grew faster than the two groups in osteogenic culture and almost completely filled the Ti-mesh voids on day 8 (Fig. 6A-C). By day 8 , the 2 cultures in osteogenic medium began to produce collagen and to form mineralized nodules. After 24 days of incubation, more numerous and larger mineralized globular accretions always associated with collagen bundles were observed (Fig. 6D, E). In the non-osteogenic cell culture, the formation of a mineralized extracellular matrix was never observed (Fig. 6F).

Expression levels of mRNA for ALP, Col I, OC, DSPP, and DMP-1 were compared on days 1, 4, 8, and 16. mRNA expression levels of non-osteogenic cell cultures on day 1 were set as baseline controls (relative expression value, $100 \%$ ). Results showed that ALP was significantly greater in transfected cells (Fig. 7A). Col I showed up-regulation for all 3 groups and was significantly higher in cells cultured in non-osteogenic medium (Fig. 7B). OC showed some up- regulation in the 2 groups cultured in osteogenic medium at days 8 and 16, but the expression level of transfected cells was significantly higher than of non-transfected cells at day 8 (Fig. 7C). The expression of DSPP and DMP-1 revealed a significantly greater number of cells cultured in osteogenic medium at days 8 and 16, although the transfected cells had a higher expression levels than the non-transfected cells (Fig. 7D, E).

\section{DISCUSSION}

Effective gene delivery into mammalian somatic cells in vitro and in vivo is an essential step in gene therapy. In this study, we have demonstrated gene transfection and a subsequent odonto-stimulatory effect in STRO-1-selected rat DPSCs through pEGFP-BMP2-loaded calcium phosphate nanoparticles. 


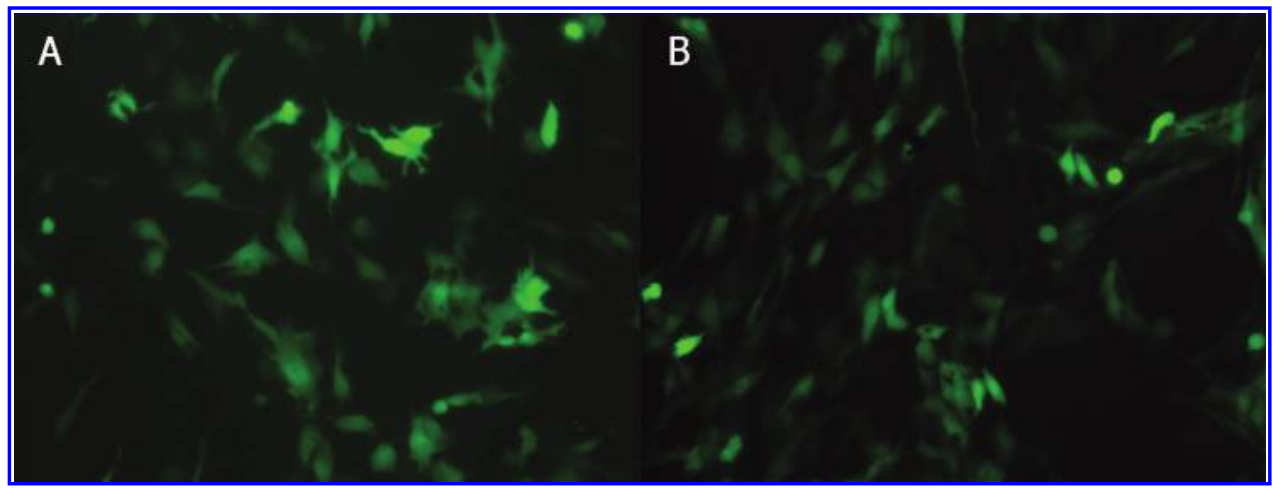

FIG. 3. Fluorescent micrographs of green fluorescent protein expression in permuted enhanced green fluorescent protein-bone morphogenetic protein 2-transfected rat dental pulp stem cells. (A) Calcium phosphate nanoparticle group; (B) Lipofectamine group (original magnification 10×). Color images available online at www.liebertpub.com/ten.

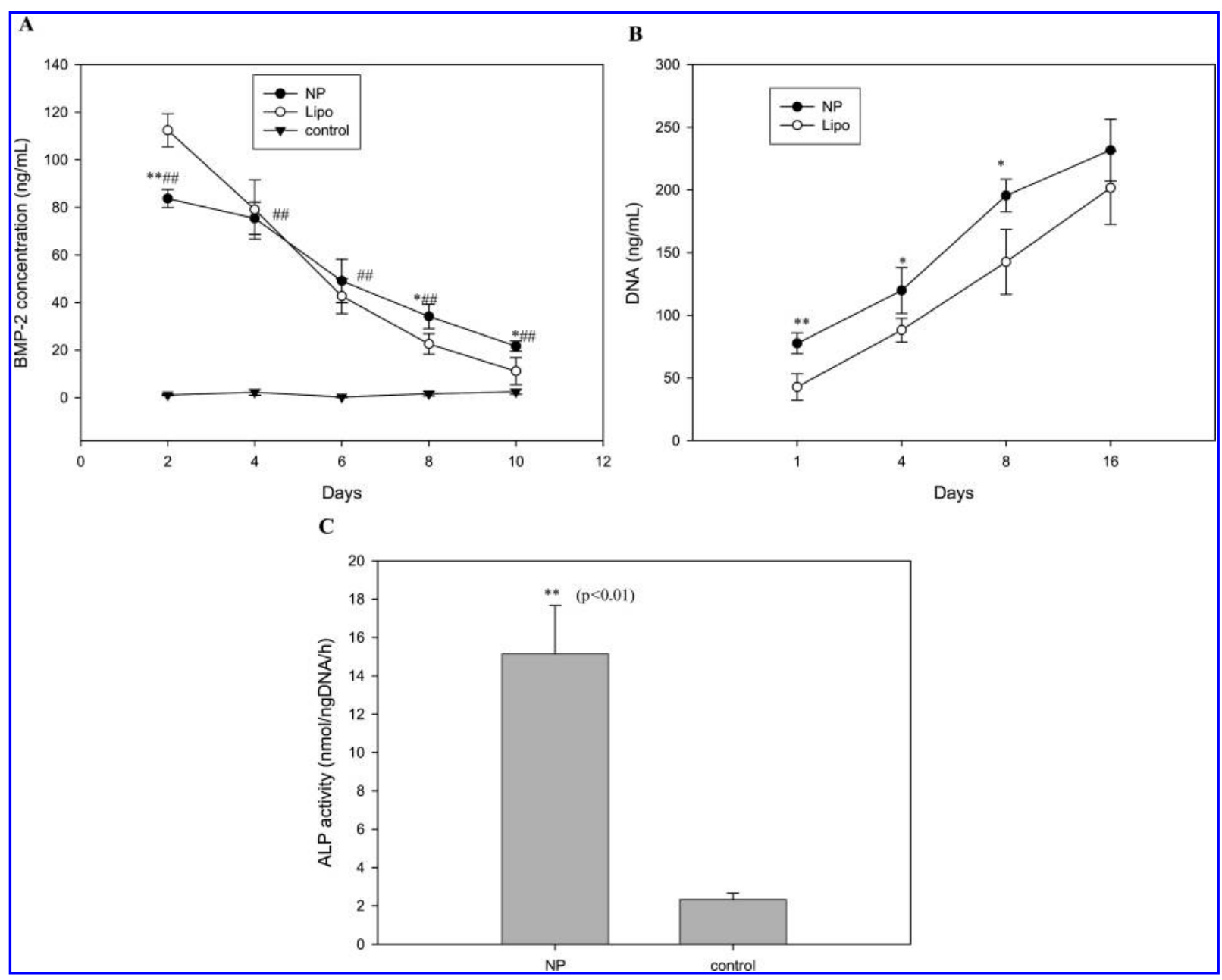

FIG. 4. (A) Enzyme-linked immunosorbent assay for human bone morphogenetic protein 2 (hBMP2) concentration in 3 groups. The bars represent means \pm standard deviations (SDs) $(n=6)$. The calcium phosphate nanoparticle group (NP) compared with the Lipofectamine group (Lipo): ${ }^{*} p<0.05,{ }^{*} p<0.01$; NP group compared with non-osteogenic group (control): \#\#p $<0.01$. (B) The proliferation characteristics of 2 groups. The bars represent means \pm SDs $(n=6)$. NP group compared with lipo group (Lipo): $* p<0.05$, $*^{*} p<0.01$. (C) The bioactivity of hBMP2 produced from transfected rat dental pulp stem cells (DPSCs) mediated with nanoparticles $48 \mathrm{~h}$ post-transfection. Bioactivity was assessed according to up-regulation of alkaline phospotase activity in W20-17 cells induced by conditional medium incubated with rat DPSCs. The bars represent the means \pm SDs $(n=6)$. NP group compared with non-osteogenic group (control): $* * p<0.01$. DNA, deoxyribonucleic acid. 


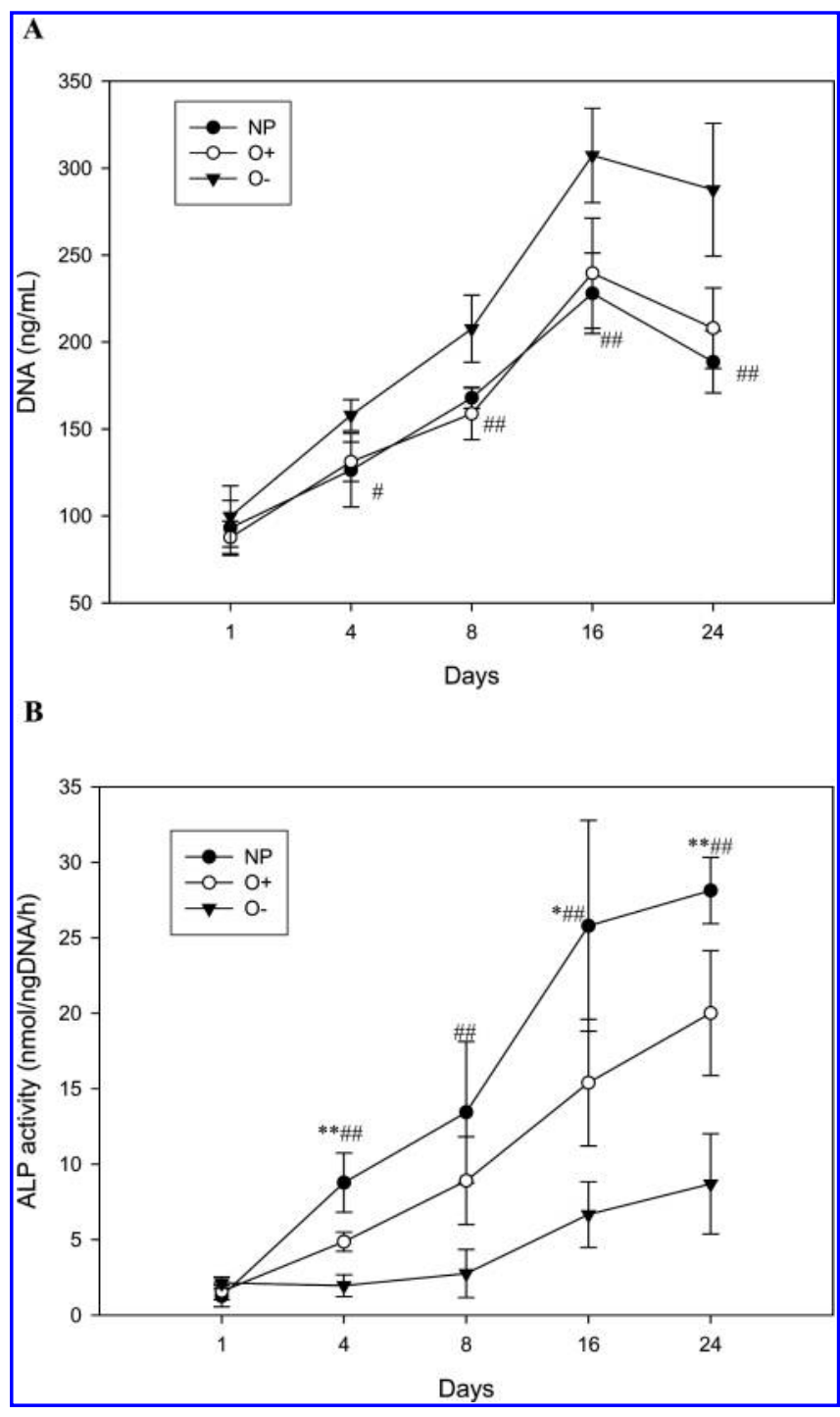

FIG. 5. (A) The proliferation characteristics; (B) alkaline phosphatase (ALP) activity; (C) calcium content. The bars represent the means \pm standard deviations $(n=3)$. The calcium phosphate nanoparticle group (NP) compared with the group in osteogenic medium $\left(\mathrm{O}^{+}\right) * p<0.05, * * p<0.01$; NP group compared with the group in non-osteogenic medium $\left(\mathrm{O}^{-}\right)$: \#p $<0.05$, \#\#p $<0.01$.

Two classical types of non-viral vectors are liposomal formulations and cationic polymers. Unfortunately, it has been observed that, in addition to cytotoxicity, these vectors do not always lead to satisfactory efficacy of gene expression in the cells. The reasons may be due to low endosomal escape, no protection of DNA from nuclease degradation, and inefficient nuclear uptake. ${ }^{18-20}$ Calcium ions are known to form ionic complexes with the helical phosphates of DNA, and these complexes have easy transportability across the cell membrane via ion channel-mediated endocytosis. ${ }^{21}$ It was envisaged that the use of nanoparticles of inorganic calcium might eliminate many of these limitations. Previous studies have reported the use of pDNA encapsulated in

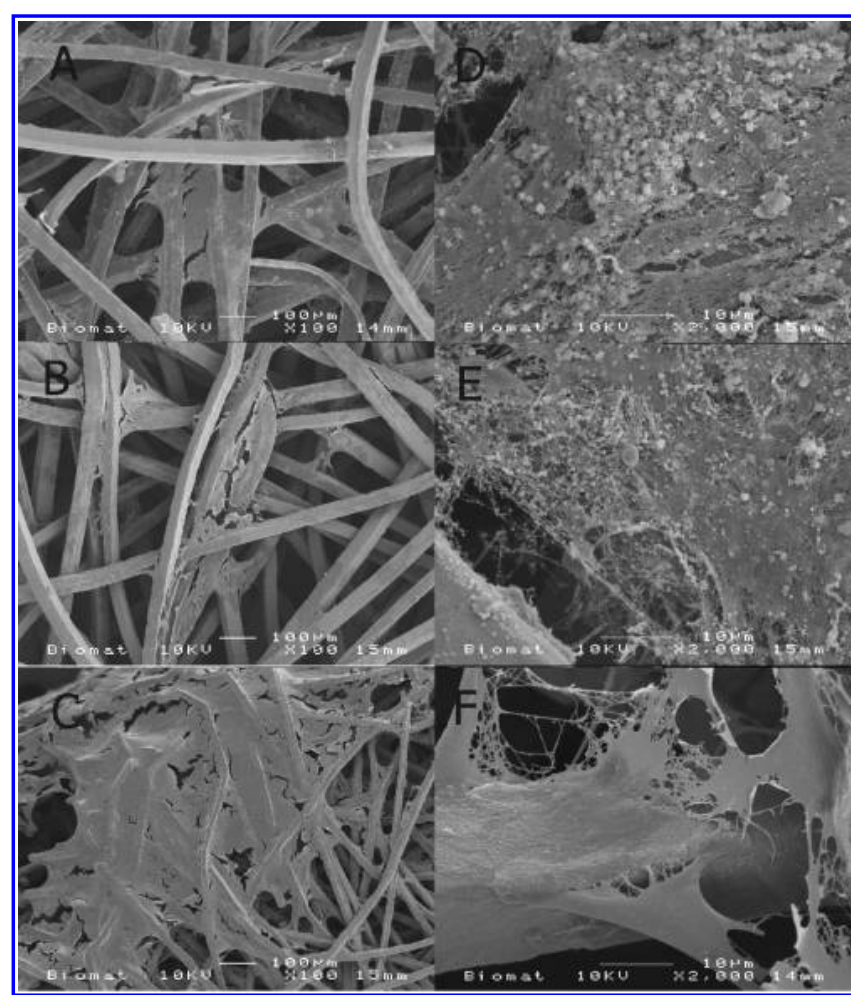

FIG. 6. Scanning electron micrographs of 3 cell populations on day 8: (A) the transfected dental pulp stem cells (DPSCs), (B) the DPSCs in osteogenic medium, $(\mathbf{C})$ the DPSCs in non-osteogenic medium, On day 24: (D) the transfected DPSCs, (E) the DPSCs in osteogenic medium, (F) the DPSCs in non-osteogenic medium.

calcium phosphate nanoparticles as gene carriers. These calcium phosphate nanoparticles have a well-defined role in DNA delivery as far as endosomal escape, protection against nuclease degradation, and nuclear uptake are concerned. ${ }^{6,7}$

The literature shows that $5.88 \mu \mathrm{g}$ of pDNA per mg of calcium phosphate can be loaded in the nanoparticles. ${ }^{6}$ Using the same ratio, we also acquired particles less than $100 \mathrm{~nm}$ in diameter. It has been confirmed that particles greater than $200 \mathrm{~nm}$ in diameter are not efficiently taken up by endocytosis and thus only via phagocytosis. ${ }^{22}$ One previous study showed that the surface charge of DNA-loaded calcium phosphate nanoparticles is positive in neutral aqueous buffer, ${ }^{6}$ resulting in non-movable nanoparticles in normal electrophoresis running toward the anode. Furthermore, the DNA encapsulated in calcium phosphate particles was protected for the external DNase environment, which was favorable for the survival of DNA after endocytosis.

The endosomal escape and the nuclear uptake are the final obstacles for DNA delivery. ${ }^{20}$ It has been demonstrated that calcium ions play an important role in endosomal escape, cytosolic stability, and enhanced nuclear uptake of DNA through nuclear pore complexes. ${ }^{23}$ The inorganic nanoparticles are necessary as DNA carriers for entry into the cell. In our case, the DNA-doped nanoparticles are 
A

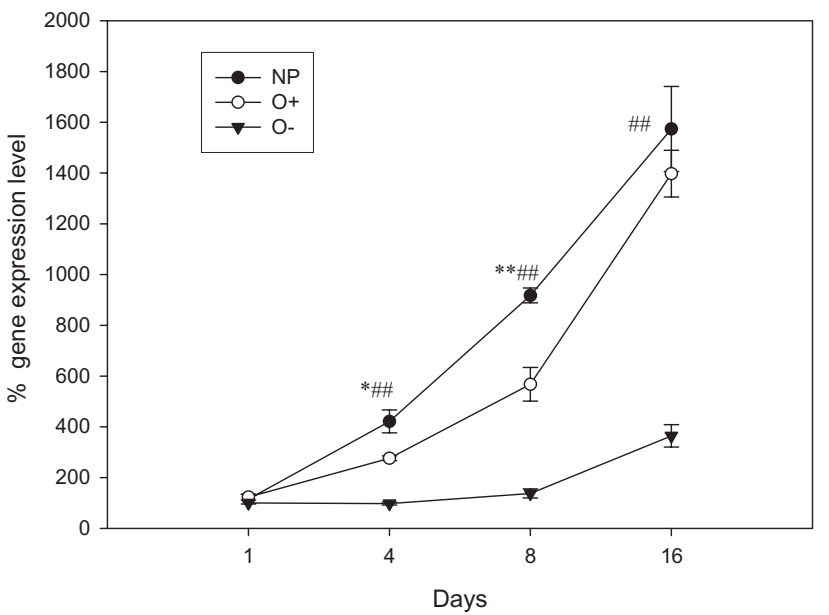

C

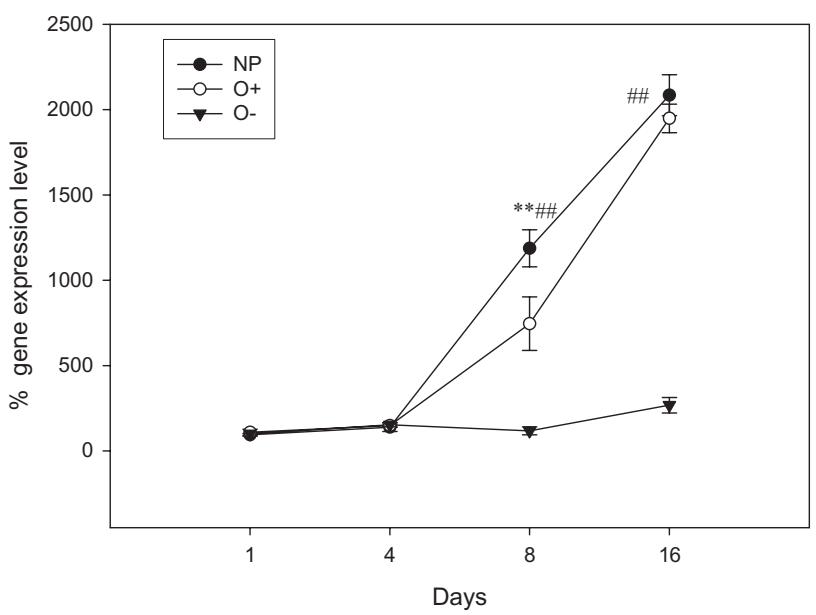

B

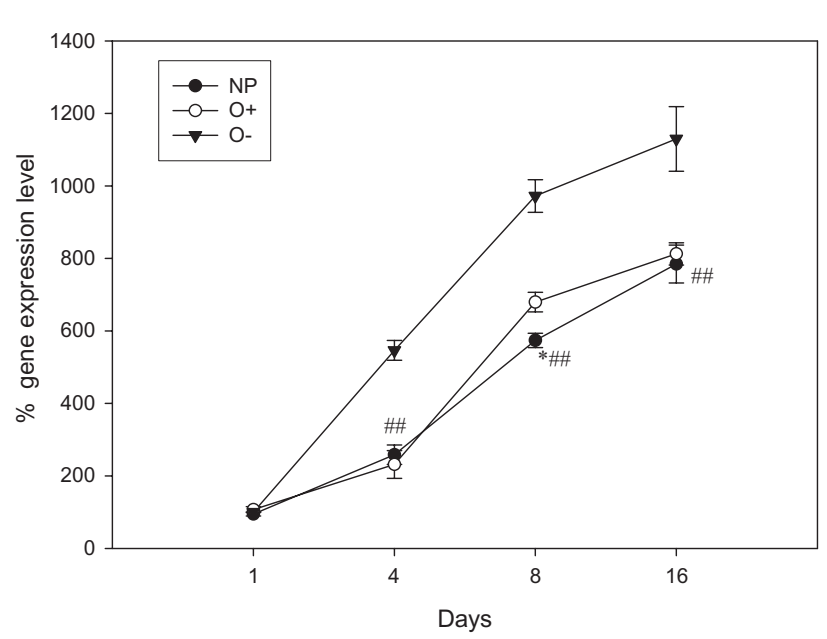

D

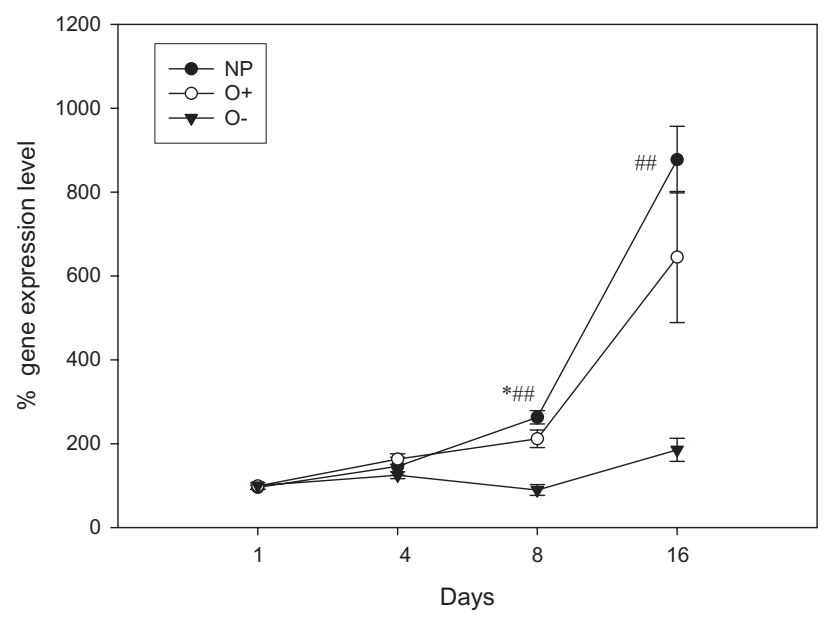

$\mathbf{E}$

DMP-1

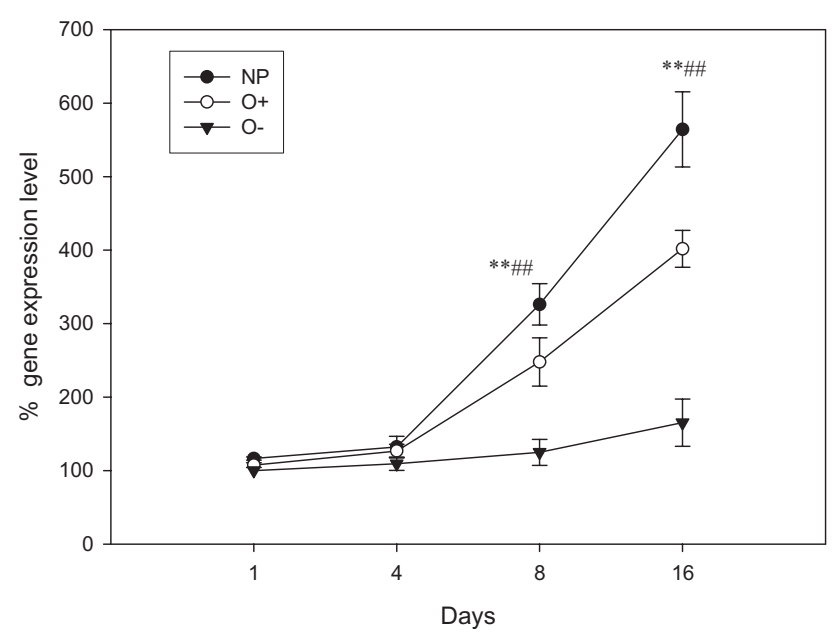

FIG. 7. Representative curves of the messenger ribonucleic acid levels of alkaline phosphatase (A); collagen type I (B); osteocalcin (C); dentin sialophosphoprotein (D); dentin matrix protein 1 (E) quantified during 16 days. The bars represent means \pm standard deviations $(n=3)$. The calcium phosphate nanoparticle group (NP) compared with the group in osteogenic medium $\left(\mathrm{O}^{+}\right) * p<0.05, * * p<0.01 ; \mathrm{NP}$ group compared with the group in non-osteogenic medium $\left(\mathrm{O}^{-}\right)$: \#p $<0.05$, \#\#p $<0.01$. 
non-crystalline, in contrast to voided calcium phosphate nanoparticles. ${ }^{6,7}$ In the present study, we observed successful gene expression from nanoparticle-mediated cells, and the gene products were bioactive. As a control, Lipofectamine-mediated cells were used, which were shown to produced higher hBMP2 levels initially, although at later time points, the nanoparticle-mediated group reached higher levels. The final amount of BMP seems to be the most interesting output parameter when subsequently going to validation (animal) studies. Differences can be the result of different methods of endosomal escape. After endocytosis, lipid-DNA compounds internalize into the endosome and initiate the destabilization of the endosomal membrane for escaping. ${ }^{20,24}$ However, the dissolution of inorganic nanoparticles in the acidic endosomal compartment ( $\mathrm{pH} 5.0$ 5.5) results in an osmotic imbalance and the consequent disruption of the endosome so that DNA can escape into the cytosol. ${ }^{6}$ There were fewer cells transfected with Lipofectamine from day 1 to 8 than in the nanoparticle group, which might have been because of the cytotoxicity of cationic liposomes. ${ }^{25}$ Altogether, Lipofectamine was advantageous for the BMP level obtained at the beginning (i.e., at day 2), but after that, the nanoparticle strategy was superior.

Titanium fiber mesh is a highly biocompatible material, and its non-degradable nature is not necessarily disadvantageous for hard-tissue applications. Previous studies have demonstrated that titanium fiber mesh, which has been used for bone regeneration, ${ }^{26}$ supportss the attachment, growth, and differentiation of dental pulp cells in vivo and in vitro. ${ }^{16,27}$ In the current study, the non-osteogenic DPSC culture proliferated faster because of the inverse relationship between proliferation and differentiation (i.e., when cells are more proliferative then the slowing in differentiation of the cells and vice versa). ${ }^{28}$ It is known that BMP2 in high concentrations can stimulate the early differentiation of cells and the formation of extracellular matrix while not influencing proliferation. ${ }^{29}$

ALP is a prerequisite for the differentiation of dental pulp cells. We observed a longer period of ALP activity than in previous studies. ${ }^{16}$ A 3-D scaffold provides a larger surface area for cell attachment than a smooth substrate, which is favorable for the spreading and interaction of cells and thus results in a longer increase in the ALP activity. ${ }^{16}$ Moreover, in our study, STRO-1-selected rat DPSCs were used, which showed earlier up-regulation of ALP activity than did nonselected rat dental pulp cells on a similar scaffold ${ }^{16}$ because of the more homogeneous cells from STRO-1 selection. ${ }^{15}$ Comparison of ALP activity between the 2 groups that were cultured in the osteogenic medium revealed that the secreted BMP2 from the nanoparticle-transfected cells exerts its effect and accelerates the odontogenic differentiation and matrix mineralization of DPSCs. Previous studies have revealed that BMP2 functions in a dose-dependent manner. High concentrations (e.g., $100 \mathrm{ng} / \mathrm{mL}$ ) of BMP2 demon- strated the induction of an osteoblastic phenotype. ${ }^{29,30}$ Our study shows that the transfected cells mediated with nanoparticles produce less than $100 \mathrm{ng} / \mathrm{mL}$ of hBMP2, although the transfected cells can continuously secrete the Bmp2 gene product into the medium, which may account for similar bio-functionality to the higher concentrations necessary with BMP2 as a protein supplement in a single application. ${ }^{31}$ Furthermore, calcium content measurement demonstrated that the transfected cells led to more mineralization, and SEM observation showed that mineralized matrix fully covered many cells. Although a previous study showed that calcium phosphate nanocomposites by themselves can induce cell differentiation and bone formation, ${ }^{32}$ we feel that the gene products were mostly responsible for cell differentiation, especially bearing in mind the low-dose calcium phosphate particles used in this study. Ideally, an empty nanoparticle would have been used as negative control, but this was not possible, because the production of non-DNAloaded particles would have resulted in different properties regarding morphology, composition, and crystallinity.

The real-time PCR further confirmed the results mentioned above. It is known that mRNA expression is always seen before protein expression. mRNA expression of ALP also continued to increase and reached a peak at day 16 for cells in osteogenic culture, whereas the transfected cells had a more-sensitive reaction. Collagen type $\mathrm{I}$ is the predominant collagen in dentin and bone matrix and the foundation of the extracellular matrix on which future mineralization takes place. Higher levels of collagen type I mRNA were found in cells from non-osteogenic culture, which was in concordance with the cell growth profiles found through DNA analysis and SEM. OC, a noncollagenous protein, is also present in dentin and bone matrix. mRNA expression of OC showed similar profiles to those found in a previous study. ${ }^{16}$ However, the two groups in osteogenic culture demonstrated differences only at day 8. This may be due to the BMP2, which stimulates the early differentiation of cells, whereas OC is known as a late-phase marker for terminal cell differentiation and matrix mineralization. ${ }^{33}$ DSPP is the major part of noncollagenous proteins in natural dentin and plays a crucial role during dentin mineralization. ${ }^{34}$ DMP- 1 is another specific protein in dentin and is a candidate gene for dentinogenesis imperfecta. ${ }^{35}$ Expression of these mRNAs can prove that these cells exhibit an odontoblast phenotype.

\section{CONCLUSIONS}

Based on our results, we concluded that rat STRO-1selected DPSCs can be successfully transfected with pEGFP-BMP2-loaded calcium phosphate nanoparticles. In some aspects, this nanoparticle vector was superior to the commercial Lipofectamine transfection reagent. The secreted BMP2 reached a functional level and accelerated 
odontogenic differentiation. Consequently, calcium phosphate nanoparticles can be considered a valid candidate as a non-viral gene vector. Meanwhile, the established combination of a more-homogeneous cell population with gene transfection can be a strategy to improve the efficacy of DPSCs for hard-tissue engineering.

\section{ACKNOWLEDGMENTS}

The authors thank Lise de Jonge for technical assistance. SEM was performed at the Microscopic Imaging Centre of the Nijmegen Centre for Molecular Life Sciences, The Netherlands. This work was supported by the Royal Netherlands Academy of Arts and Sciences (KNAW; 06CDP030).

\section{REFERENCES}

1. Bonadio J, Smiley E, Patil P, et al. Localized, direct plasmid gene delivery in vivo: prolonged therapy results in reproducible tissue regeneration. Nat Med. 5, 753, 1999.

2. Wozney JM, Rosen V. Bone morphogenetic protein and bone morphogenetic protein gene family in bone formation and repair. Clin Orthop Relat Res. 26, 1998.

3. Yang XC, van den Dolder J, Walboomers XF, et al. STRO-1 selected rat dental pulp stem cells transfected with adenoviralmediated human Bmp2 gene. Tissue Eng. 2007 Sep 9; [Epub ahead of print].

4. Ferber D. Gene therapy. Safer and virus-free? Science. 294, 1638, 2001.

5. Somia N, Verma IM. Gene therapy: trials and tribulations. Nat Rev Genet. 1, 91, 2000.

6. Roy I, Mitra S, Maitra A, et al. Calcium phosphate nanoparticles as novel non-viral vectors for targeted gene delivery. Int J Pharm. 250, 25, 2003.

7. Bisht S, Bhakta G, Mitra S, et al. pDNA loaded calcium phosphate nanoparticles: highly efficient non-viral vector for gene delivery. Int J Pharm. 288, 157, 2005.

8. Boden SD, Zdeblick TA, Sandhu HS, et al. The use of rhBMP-2 in interbody fusion cages. Definitive evidence of osteoinduction in humans: a preliminary report. Spine. 25, 376, 2000.

9. Hogan BL. Bone morphogenetic proteins in development. Curr Opin Genet Dev. 6, 432, 1996.

10. Thesleff I, Sharpe P. Signaling networks regulating dental development. Mech Dev. 67, 111, 1997.

11. Iohara K, Nakashima M, Ito M, et al. Dentin regeneration by dental pulp stem cell therapy with recombinant human bone morphogenetic protein 2. J Dent Res. 83, 590, 2004.

12. Gronthos S, Mankani M, Brahim J, et al. Postnatal human dental pulp stem cells (DPSCs) in vitro and in vivo. Proc Natl Acad Sci U S A. 97, 13625, 2000.

13. Gronthos S, Brahim J, Li W, et al. Stem cell properties of human dental pulp stem cells. J Dent Res. 81, 531, 2002.

14. Yang XC, van den Dolder J, Walboomers XF, et al. Multilineage potential of STRO- $1^{+}$dental pulp stem cells in vitro. J Tissue Eng Regen Med 1, 128, 2007.
15. Laino G, Graziano A, d'Aquino R, et al. An approachable human adult stem cell source for hard-tissue engineering. J Cell Physiol. 206, 693, 2006.

16. Zhang W, Walboomers XF, Wolke JG, et al. Differentiation ability of rat postnatal dental pulp cells in vitro. Tissue Eng. 11, 357, 2005.

17. Thies RS, Bauduy M, Ashton BA, et al. Recombinant human bone morphogenetic protein-2 induces osteoblastic differentiation in W20-17 stromal cells. Endocrinology. 130, 1318, 1992.

18. Cullis PR, Chonn A. Recent advances in liposome technologies and their applications for systemic gene delivery. Adv Drug Deliv Rev. 30, 73, 1998.

19. Ramsay E, Hadgraft J, Birchall J, et al. Examination of the biophysical interaction between plasmid DNA and the polycations, polylysine and polyornithine, as a basis for their differential gene transfection in-vitro. Int J Pharm. 210, 97, 2000.

20. Luo D, Saltzman WM. Synthetic DNA delivery systems. Nat Biotechnol. 18, 33, 2000.

21. Orrantia E, Chang PL. Intracellular distribution of DNA internalized through calcium phosphate precipitation. Exp Cell Res. 190, 170, 1990.

22. Mahato RI, Rolland A, Tomlinson E. Cationic lipid-based gene delivery systems: pharmaceutical perspectives. Pharm Res. 14, 853, 1997.

23. Maitra A. Calcium phosphate nanoparticles: second-generation nonviral vectors in gene therapy. Expert Rev Mol Diagn. 5, 893, 2005.

24. El Ouahabi A, Thiry M, Pector V, et al. The role of endosome destabilizing activity in the gene transfer process mediated by cationic lipids. FEBS Lett. 414, 187, 1997.

25. Hirko A, Tang F, Hughes JA. Cationic lipid vectors for plasmid DNA delivery. Curr Med Chem. 10, 1185, 2003.

26. van den Dolder J, Farber E, Spauwen $\mathrm{PH}$, et al. Bone tissue reconstruction using titanium fiber mesh combined with rat bone marrow stromal cells. Biomaterials. 24, 1745, 2003.

27. Zhang W, Frank Walboomers X, van Kuppevelt TH, et al. The performance of human dental pulp stem cells on different threedimensional scaffold materials. Biomaterials. 27, 5658, 2006.

28. van den Dolder J, Spauwen PH, Jansen JA. Evaluation of various seeding techniques for culturing osteogenic cells on titanium fiber mesh. Tissue Eng. 9, 315, 2003.

29. van den Dolder J, de Ruijter AJ, Spauwen PH, et al. Observations on the effect of BMP-2 on rat bone marrow cells cultured on titanium substrates of different roughness. Biomaterials. 24, 1853, 2003.

30. Vehof JW, de Ruijter AE, Spauwen PH, et al. Influence of rhBMP-2 on rat bone marrow stromal cells cultured on titanium fiber mesh. Tissue Eng. 7, 373, 2001.

31. Wozney JM, Rosen V. Bone morphogenetic protein and bone morphogenetic protein gene family in bone formation and repair. Clin Orthop Relat Res. 26, 1998.

32. Lin Y, Wang T, Wu L, et al. Ectopic and in situ bone formation of adipose tissue-derived stromal cells in biphasic calcium phosphate nanocomposite. J Biomed Mater Res A. 81, 900, 2007.

33. Dragoo JL, Choi JY, Lieberman JR, et al. Bone induction by BMP-2 transduced stem cells derived from human fat. J Orthop Res. 21, 622, 2003. 
34. Feng JQ, Luan X, Wallace J, et al. Genomic organization, chromosomal mapping, and promoter analysis of the mouse dentin sialophosphoprotein (Dspp) gene, which codes for both dentin sialoprotein and dentin phosphoprotein. $\mathrm{J}$ Biol Chem. 273, 9457, 1998.

35. MacDougall M, Gu TT, Simmons D. Dentin matrix protein-1, a candidate gene for dentinogenesis imperfecta. Connect Tissue Res. 35, 267, 1996.
Address reprint requests to: John A. Jansen, D.D.S., Ph.D. P.O. Box 9101 6500 HB Nijmegen The Netherlands

E-mail: J.Jansen@dent.umcn.nl 



\section{This article has been cited by:}

1. T. Srisuwan, D.J. Tilkorn, S. Al-Benna, A. Vashi, A. Penington, H.H. Messer, K.M. Abberton, E.W. Thompson. 2012. Survival of rat functional dental pulp cells in vascularized tissue engineering chambers. Tissue and Cell . [CrossRef]

2. Erica G. Tierney, Garry P. Duffy, Alan J. Hibbitts, Sally-Ann Cryan, Fergal J. O'Brien. 2011. The development of nonviral gene-activated matrices for bone regeneration using polyethyleneimine (PEI) and collagen-based scaffolds. Journal of Controlled Release . [CrossRef]

3. Cai-Xia He, Ni Li, Yu-Lan Hu, Xiu-Mei Zhu, Hai-Jie Li, Min Han, Pei-Hong Miao, Zhong-Jie Hu, Gang Wang, Wen-Quan Liang, Yasuhiko Tabata, Jian-Qing Gao. 2011. Effective Gene Delivery to Mesenchymal Stem Cells Based on the Reverse Transfection and Three-Dimensional Cell Culture System. Pharmaceutical Research 28:7, 1577-1590. [CrossRef]

4. Mathilde Roger, Anne Clavreul, Marie-Claire Venier-Julienne, Catherine Passirani, Claudia Montero-Menei, Philippe Menei. 2011. The potential of combinations of drug-loaded nanoparticle systems and adult stem cells for glioma therapy. Biomaterials 32:8, 2106-2116. [CrossRef]

5. Sunyoung Nam, Jong-Eun Won, Cheol-Hwan Kim, Hae-Won Kim. 2011. Odontogenic Differentiation of Human Dental Pulp Stem Cells Stimulated by the Calcium Phosphate Porous Granules. Journal of Tissue Engineering 2011, 1-10. [CrossRef]

6. Jons Hilborn. 2011. In vivo injectable gels for tissue repair. Wiley Interdisciplinary Reviews: Nanomedicine and Nanobiotechnology n/a-n/a. [CrossRef]

7. Sergey V. Dorozhkin. 2010. Nanosized and nanocrystalline calcium orthophosphates. Acta Biomaterialia 6:3, 715-734. [CrossRef] 